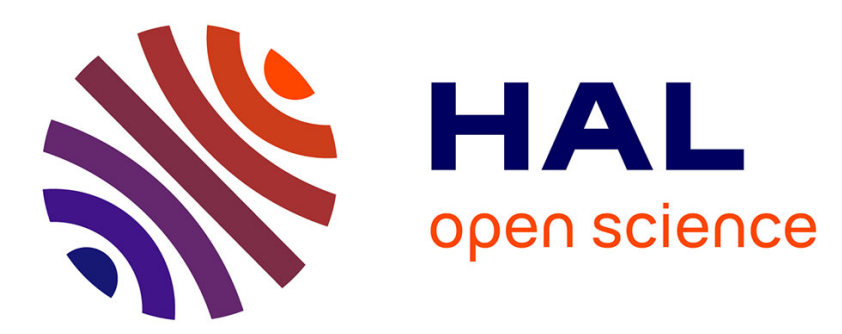

\title{
Metallic Species in Mercury's Exosphere: EMMI/New Technology Telescope Observations
}

\author{
Alain Doressoundiram, François Leblanc, C. Foellmi, S. Erard
}

\section{To cite this version:}

Alain Doressoundiram, François Leblanc, C. Foellmi, S. Erard. Metallic Species in Mercury's Exosphere: EMMI/New Technology Telescope Observations. The Astronomical Journal, 2009, 137 (4), pp.3859-3863. 10.1088/0004-6256/137/4/3859 . hal-00367621

\section{HAL Id: hal-00367621 \\ https://hal.science/hal-00367621}

Submitted on 19 May 2019

HAL is a multi-disciplinary open access archive for the deposit and dissemination of scientific research documents, whether they are published or not. The documents may come from teaching and research institutions in France or abroad, or from public or private research centers.
L'archive ouverte pluridisciplinaire $\mathbf{H A L}$, est destinée au dépôt et à la diffusion de documents scientifiques de niveau recherche, publiés ou non, émanant des établissements d'enseignement et de recherche français ou étrangers, des laboratoires publics ou privés. 


\title{
METALLIC SPECIES IN MERCURY'S EXOSPHERE: EMMI/NEW TECHNOLOGY TELESCOPE OBSERVATIONS*
}

\author{
A. Doressoundiram ${ }^{1}$, F. Leblanc ${ }^{2}$, C. Foellmi $^{3}$, and S. ERARd ${ }^{1}$ \\ ${ }^{1}$ LESIA, Observatoire de Paris, F-92195 Meudon Principal Cedex, France; alain.doressoundiram@obspm.fr \\ ${ }^{2}$ Service d'Aéronomie, Université Versailles Saint Quentin, CNRS, France \\ ${ }^{3}$ Laboratoire d'Astrophysique de l'Observatoire de Grenoble, F-38041 Grenoble Cedex 09, France \\ Received 2008 October 28; accepted 2009 January 13; published 2009 March 6
}

\begin{abstract}
We performed high spectral resolution observations of Mercury's exosphere on 2005 October 30 and 31 using the European Southern Observatory-New Technology Telescope, La Silla, Chile. The large spectral range, 385-855 nm, of the spectrograph, ESO Multi-Mode Instrument, provides a unique opportunity to search for nonidentified species in Hermian's environment. In this paper, we report a tentative detection of atomic aluminum in the exosphere of Mercury. This detection should be confirmed by further observations and can be used as an upper limit for this element in Mercury's exosphere. We also estimate the upper limit for the column densities of $\mathrm{Fe}$ and $\mathrm{Si}$ exospheric atoms. Detection of $\mathrm{Al}$, a refractory element, if confirmed, as well as its high exospheric abundance (between 2 and 18) with respect to $\mathrm{Ca}$ would suggest either an unexpected surface composition or a relation between exosphere and surface composition that is not well understood.
\end{abstract}

Key words: planets and satellites: individual (Mercury)

\section{INTRODUCTION}

Mercury is surrounded by a very tenuous atmosphere, with a maximum dayside density of about $10^{7}$ atoms $\mathrm{cm}^{-3}$ (Broadfoot et al. 1976). This atmosphere is collisionless (i.e., the mean free path of the atoms is greater than the scale height of the atmosphere) and therefore comparable to an exosphere having the exobase coincident with the planet's surface. The existence of an atmosphere around Mercury was discovered for the first time by the Mariner 10 spectrometers that revealed three atomic elements: H, He, and O (Broadfoot et al. 1976). The presence of $\mathrm{Na}, \mathrm{K}$, and $\mathrm{Ca}$ was discovered later using ground-based telescopes (Potter \& Morgan 1985, 1986; Bida et al. 2000).

The lifetime for the neutral species in Mercury's exosphere is governed by their interaction with the surface and their loss into the interplanetary medium, photoionization being the fastest loss mechanism. To maintain the detected exosphere, the lost atoms must be replaced by a source mechanism. Processes of endogenic and exogenic origins act to repopulate the exosphere: implantation and re-ejection of solar wind ions (in the case of $\mathrm{H}$ and $\mathrm{He})$, impact vaporization of meteoroids and regolith $(\mathrm{Na}$, $\mathrm{O}, \mathrm{K}, \mathrm{Ca})$, photosputtering and photodesorption $(\mathrm{Na}, \mathrm{K})$, and ion sputtering $(\mathrm{Ca}, \mathrm{Na}, \mathrm{K}, \mathrm{O})$.

In this paper, we describe observations obtained at European Southern Observatory (ESO)-New Technology Telescope (NTT) telescope with the ESO Multi-Mode Instrument (EMMI) spectrograph (Section 2) whose large spectral range (385$855 \mathrm{~nm})$ and high spectral resolution capabilities provide a unique opportunity to search for nonidentified species in Mercury's exosphere (Section 3). These results are discussed in Section 4.

\section{OBSERVATIONS AND ANALYSIS}

Observations of Mercury were obtained using the EMMI instrument at the $3.6 \mathrm{~m}$ NTT (La Silla, Chile), during the evenings of 2005 October 30 and 31 . We used the REMD mode

\footnotetext{
* Based on Observations carried out at the European Southern Observatory (ESO), La Sill, Chile, Programme id 076.c-0619(A).
}

of EMMI for high-resolution echelle spectroscopy. We used the grating echelle \#14 with the cross disperser \#3, together with a $1 \times 1$ binning and a slit of $6^{\prime \prime}$ length and 0 ".8 width. This instrumental setup yielded a spectral resolution of 75600 at central wavelength $\lambda=620 \mathrm{~nm}$ (dispersion of $\sim 0.025 \AA \mathrm{pix}^{-1}$ ) and covered the spectral range of $385-855 \mathrm{~nm}$, spanned on 89 orders. The ThAr lamp was used as spectral calibration.

The observations started as soon as the sky background was low enough so that Mercury could be seen in the guider, and ended at around airmass 5 (the telescope's lowest pointing limit is $12^{\circ}$ ). That is, we acquired data from 23:30:32 to 23:48:53 UT on October 30 and between 23:35:22 and 23:54:02 on October 31. On the first night (respectively the second night), Mercury's true anomaly angle (TAA) was equal to $227^{\circ}$ $\left(230^{\circ}\right)$, the phase angle to $66^{\circ}\left(68^{\circ}\right)$, and Mercury's diameter to $6^{\prime \prime} .2\left(6^{\prime \prime} .3\right)$. Mercury's heliocentric distance was equal to 0.428 $\mathrm{AU}$ (0.423 UA) and its heliocentric velocity was equal to $-7.71 \mathrm{~km} \mathrm{~s}^{-1}\left(-8.07 \mathrm{~km} \mathrm{~s}^{-1}\right)$. We aligned the slit with the parallactic angle in order to alleviate the atmospheric differential refraction. Tracking mode and motion of the slit with respect to Mercury's disk was done automatically. Mercury's exosphere was scanned at 16 slit positions with $20 \mathrm{~s}$ exposure time, with the slit moved perpendicularly. See Table 1 for the observational circumstances.

For the data reduction, we used standard IRAF tasks of the "echelle" module. Since the telescope was very low above the horizon, the guiding of the telescope was probably not ideal. Moreover, the very high airmass introduce a significant difference in flux between individual images. This is why we decided to extract the spectra individually from each of the two-dimensional exposures, and not from their sum.

First, the multiextension FITS files were recombined into single images. We then corrected for the bias level. Using the standard star observation of the second night, we defined a trace pattern of the echelle orders across the CCD with a width as large as possible. With the standard star image, we managed to trace 89 echelle orders. Using this trace on the combined flatfield images, a master flat field has been obtained and applied 

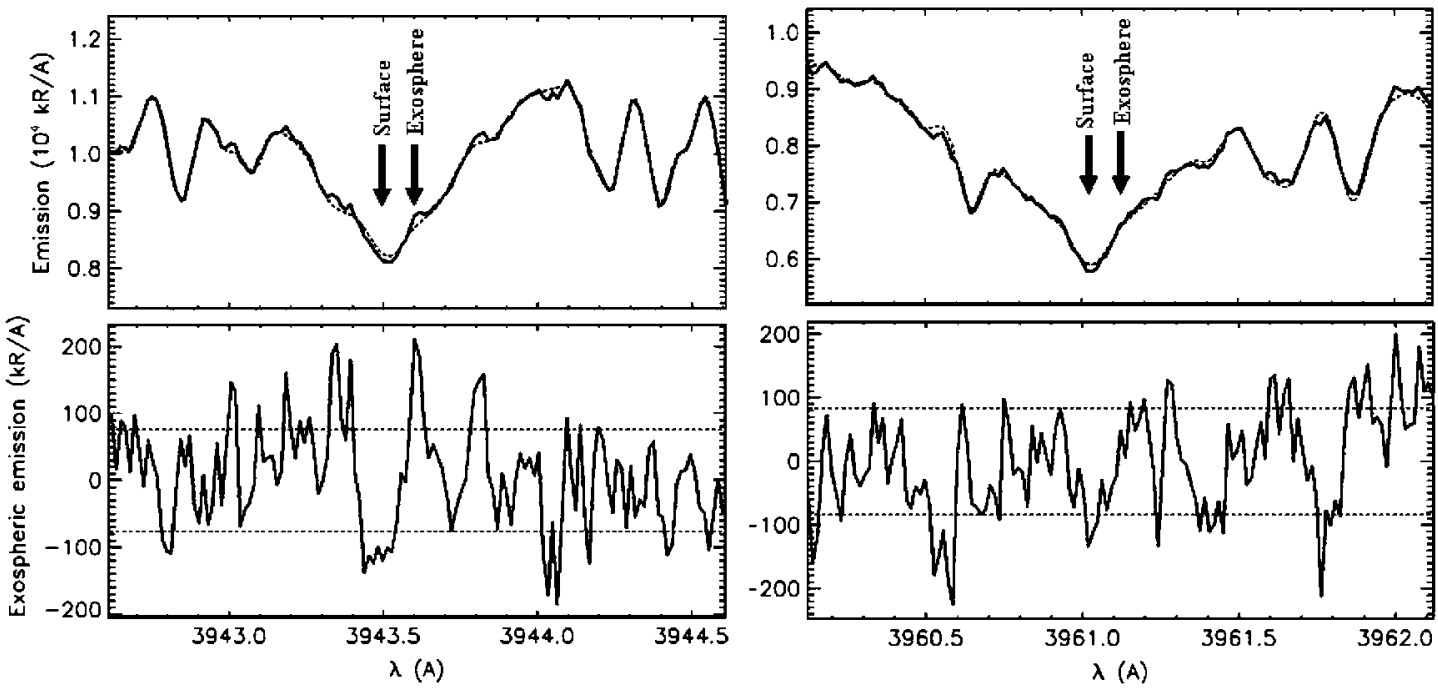

Figure 1 Spectra of the Al $3961 \AA$ (left upper panel) and $3944 \AA$ (right upper panel) resonant emission line as measured in Mercury's exosphere. The positions of the exospheric line and of the solar absorption line reflected at Mercury's surface as expected from ephemeris are also indicated. A solar spectrum (BASS 2000, http://bass2000.obspm.fr) has been reduced in resolution, scaled to the measured spectra and plotted in dashed line. Left and right lower panels are respectively the result of the subtraction of the measured spectra by the BASS 2000 spectrum. The dashed horizontal lines on the lower panels indicate the $1 \sigma$ level.

Table 1

Observational Circumstances

\begin{tabular}{lcccccc}
\hline \hline UT Date & UT Start & Heliocentric Distance (AU) & Heliocentric Velocity $\left(\mathrm{km} \mathrm{s}^{-1}\right)$ & TAA (deg) & Phase Angle (deg) & Angular Diameter (arcsec) \\
\hline 2005 Oct 30 & $23: 30: 32$ & 0.428 & -7.71 & 227 & 66 & 6.2 \\
2005 Oct 31 & $23: 35: 22$ & 0.423 & -8.07 & 230 & 68 & 6.3
\end{tabular}

to the object images. Given the EMMI instrument setup, the separation between echelle orders decreases when going toward the blue. For a pointlike object it is not a problem. However, for an elongated and very bright object such as Mercury, it makes the blue orders to overlap.

As a first consequence, we failed to extract the first four (sometimes six or seven) blue orders on some Mercury images. Those frames were discarded. For the other images, we fitted Gaussians on the horizontal cuts across the images, corresponding to the spatial profile of the orders (which are almost vertical but not perfectly, and slightly curved - this has, however, no significant influence here). Using these Gaussians, we estimate the contamination on the 5 th order to be of about $50 \%$, and rapidly decreasing.

Using the traces of extracted spectra, we obtained the corresponding Thorium-Argon calibration spectra, from images taken before the night with the exact same setup. In total, more than 400 lines were identified across the 89 echelle orders, using the atlas from La Silla Observatory completed by personal improvements since C.F. is a former La Silla Astronomer. A great care has been taken to identify the lines in the blue range. However, this task is very difficult in this domain of the spectrum given the sparse amount of lines and their weak intensities.

We also attempted a flux calibration by using a mean standard star spectrum from the second night. In order to calibrate in the flux, it was necessary to construct a one-dimensional spectrum of the standard star. For that we used the master flat to define a blaze function, which has been then divided out of the standard star spectrum. Once the orders were merged, the standard star spectrum has been used to create the sensitivity function to calibrate Mercury spectra. However, given the very high airmass of Mercury's observations, the presence of only one standard star observation, only during the second night, at a single airmass quite different from that of Mercury, we roughly estimate the flux within a factor of 2

\section{EMISSION INTENSITY MEASURED BY EMMI}

All the useful single slit images have been combined to increase the signal-to-noise ratio. Figure 1 displays a portion of the spectrum measured by the EMMI during the night of October 30. Figures 1(a) and (b) are centered on the two Al resonant emissions at 3944.0060 $\AA$ and $3961.5201 \AA$ in air (Morton $2003,2004)$. These emissions are associated with electronic transition from ${ }^{2} \mathrm{~S}$ excited state to ground state ${ }^{2} \mathrm{P}_{\frac{1}{2}}$ and ${ }^{2} \mathrm{P}_{3 / 2}$ for $3944.0060 \AA$ and $3961.5201 \AA$, respectively. These are the strongest resonant emission lines (with respect to other Al lines) within the spectral range of EMMI (NIST database, Morton et al. 2003).

We did not subtract a sky contribution to these spectra. However the comparison between the solar spectrum (BAAS 2000), dashed line in Figure 2, and the measured emission clearly shows that the contribution of the sky to the measured emission is negligible.

Taking into account the Doppler shift of the Mercury with respect to the Sun $\left(-7.71 \mathrm{~km} \mathrm{~s}^{-1}\right)$ and the relative velocity between the Earth and Mercury $\left(-30.14 \mathrm{~km} \mathrm{~s}^{-1}\right)$, we have indicated the positions of the solar emission line reflected from Mercury's surface (indicated by the arrow with "Surface") and of the exospheric emission line (indicated by the arrow with "exosphere") in Figure 1. As can be seen in the left upper panel, there is a peak in the measured spectra at the expected position for the 3944.0060 A line. Using the solar spectrum (BAAS 2000) convolved with the instrumental function to subtract the reflected light on the Mercury, it is possible to extract any potential emission lines (lower panels in Figure 1). As shown in the left lower 

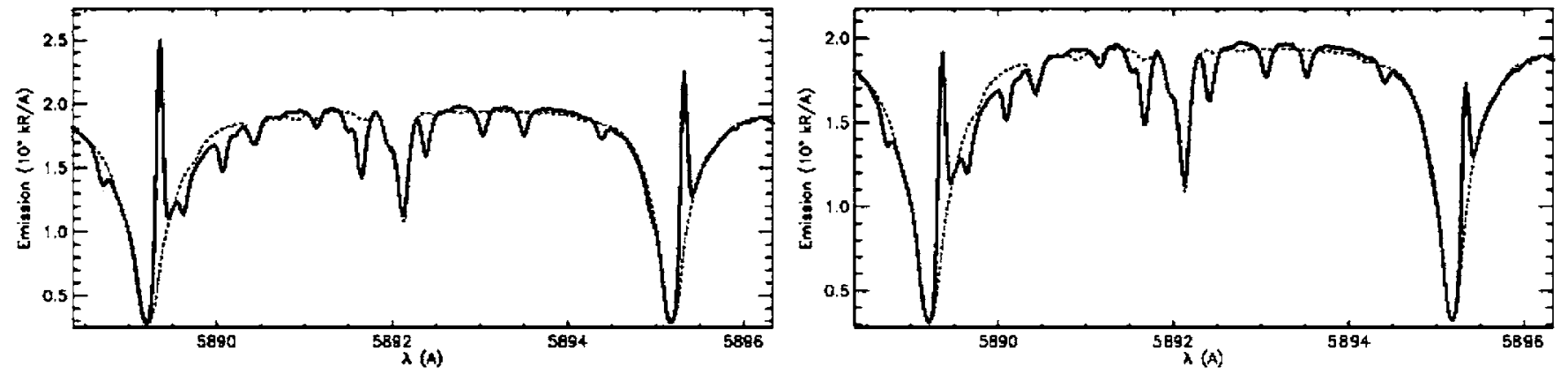

Figure 2 Spectrum measured by EMMI/NTT centered on the $\mathrm{Na}_{1}$ and $\mathrm{D}_{2}$ emission lines. The positions of the exospheric line and of the solar absorption line reflected at Mercury's surface as expected from ephemeris are also indicated. A solar spectrum (BASS 2000, http://bass2000.obspm.fr) has been reduced in resolution, scaled to the measured spectra and plotted in dashed line. Left panel: measured during the night of October 30. Right panel: measured during the night of October 31 .

panel, the $3944.0060 \AA$ emission line peaked at 2.8 standard deviation $(\sigma)$. There are "emission peaks" at 3943.8, 3943.35, and $3943.0 \AA$ that are just weaker than the apparent aluminum emission peak. However, the 3943.35 and 3943.0 peaks are too narrow to be due to emission lines. The 3943.8 line appears weaker $(<2 \sigma)$. In contrast, we did not identify a clear emission line corresponding to the $3961.5201 \AA$ emission line, except a small bump peaking at $1.2 \sigma$. It is still possible to integrate these two lines. We found intensities of $10.7 \pm 9.1 \mathrm{kRayleigh}(\mathrm{kR})$ and $6.0 \pm 8.7 \mathrm{kR}$ for the $3944.0060 \AA$ and $3961.5201 \AA$, respectively. The full width at half-maximum (FWHM) of the $3944.0060 \AA$ emission line is $\sim 40 \mathrm{~m} \AA$ whereas the FWHM of the bright $\mathrm{Na}$ $\mathrm{D}_{1}$ and $\mathrm{D}_{2}$ emission lines is equal to $90 \mathrm{~m} \AA$, also to be compared to the $78 \mathrm{~m} \AA$ instrumental resolution. Such a discrepancy might be due to the poor signal-to-noise ratio of the $3944.0060 \AA$ emission line and to the difficulty in properly extract the emission line. It is therefore not possible to firmly conclude on the detection of the $\mathrm{Al}$ emission line in Mercury's exosphere. Nonetheless, the measured intensity derived from this observation can be used as an upper limit for this element in Mercury's exosphere. Moreover, if confirmed by further observations, we would like, in the following, to highlight what could be deduced on Mercury's exosphere formation from such a type of observation.

We did not observe any emission line during the second night of observation. However, when comparing the $\mathrm{Na} \mathrm{D}_{1}$ and $\mathrm{D}_{2}$ emission lines for both nights (Figure 2), it can be seen that these emissions were significantly less bright during the second night when compared to the continuum. Therefore, either during the first night the exosphere of Mercury (at least its sodium component) was particularly bright or during the second night Mercury's exospheric sodium component was particularly less bright. The $\mathrm{Na} \mathrm{D}_{2}$ and $\mathrm{D}_{1}$ emission brigthnesses are of $1710 \pm 127 \mathrm{kR}$ and $1260 \pm 127$, respectively, during the first night which is significantly larger than the $1110 \pm$ 112 and $730 \pm 98 \mathrm{kR}$ emission brigthnesses for the second night.

Below we calculate an equivalent column density for these emission intensities under the assumption that the exosphere is optically thin. In that case, the column density $N$ along the line of sight (in atoms $\mathrm{cm}^{-2}$ ) is related to the emission intensity $4 \Pi \times \mathrm{I}$ in Rayleigh by the following relation $4 \Pi \times \mathrm{I}=10^{-6} \mathrm{~N} \times \mathrm{g}$ where $g\left(\right.$ in s $^{-1}$ ) is the photon scattering probability of $\mathrm{Al}$ atoms at Mercury orbit. $g$ depends on the solar flux at the emission line calculated at Mercury's distance to the Sun and on the absorption oscillator strength. Using a high-resolution visible solar flux measured by Kurucz et al. (1984), the absorption oscillator strengths given by Morton $(2003,2004)$ and taking into account Mercury's Doppler shift with respect to the Sun, we have calculated $g$ at $1 \mathrm{AU}$ as being equal to $0.144 \mathrm{~s}^{-1}$ for the $3944.0060 \AA$ A emission line and of $0.141 \mathrm{~s}^{-1}$ for the $3961.5201 \AA$ emission line. During the first night of EMMI observations, Mercury was at a heliocentric distance of 0.428 AU so that $g$ IN the Mercury was equal to $0.786 \mathrm{~s}^{-1}$ for the $3944.0060 \AA$ emission line and to $0.770 \mathrm{~s}^{-1}$ for the $3961.5201 \AA$ emission line.

Therefore, we found column densities equal to $14 \pm 11 \times 10^{9}$ $\mathrm{Al} \mathrm{cm}{ }^{-2}$ and $7.8 \pm 11 \times 10^{9} \mathrm{Al} \mathrm{cm}^{-2}$ when calculated from the $3944.0060 \AA$ and $3961.5201 \AA$ emission lines, respectively.

Morgan \& Killen (1997) have predicted a value for the zenith column density of $3 \times 10^{9} \mathrm{Al} \mathrm{cm}^{-2}$ which is equivalent to $4.2 \times 10^{10} \mathrm{Al} \mathrm{cm}{ }^{-2}$ along a tangent line of sight (Morgan and Killen regolith model) and zenith column densities $4.5 \times$ $10^{9} \mathrm{Al} \mathrm{cm}{ }^{-2}, 1.2 \times 10^{9} \mathrm{Al} \mathrm{cm}^{-2}$, and $1.43 \times 10^{11}$, for the volatile, intermediate, and refractory models of Goettel et al. (1988). These should be multiplied by $\sim 14$ to derive tangent column density following Morgan \& Killen (1997). Knowing the present uncertainty on our measured column densities, the volatile and intermediate models theoretical numbers are in good agreement with our measured ones.

The Na D2 and D1 emission brigthnesses of the first night correspond to column densities between $7.4 \pm 0.5 \times 10^{10} \mathrm{Na}$ $\mathrm{cm}^{-2}$ and $9.2 \pm 0.9 \times 10^{10} \mathrm{Na} \mathrm{cm}^{-2}$ which are slightly larger than the column density between 2 and $6 \times 10^{10} \mathrm{Na} \mathrm{cm}^{-2}$ estimated by Potter et al. (2007) for a similar TAA. The Ca emission line at a wavelength of 4226.728 in air (Morton 2003) has been discovered by Bida et al. (2000) using the Keck telescope and spatially and spectrally described by Killen et al. (2005). We have looked for this emission line in the EMMI spectrum but did not succeed in identifying such an emission (Figure 3).

We can however calculate an upper limit for the intensity of this emission line. The standard deviation between solar flux and measured flux around the $\mathrm{Ca}$ emission line is equal to $\sigma=64 \mathrm{kR} \AA^{-1}$, the FWHM of a spectral line is equal to 90 $\mathrm{m} \AA$ (estimated from the D2 and D1 Na emission lines, Figure 2) and therefore the integration of a Gaussian function of peak value equal to three standard deviation, and of FWHM equal to $\lambda=90 \mathrm{~m} \AA$ is equal to $3 \times \sigma \times \lambda \times \Pi^{1 / 2} /\left(2 \times(\ln (2))^{1 / 2}\right)$ $=19 \mathrm{kR}$. Bida et al. (2000) reported emission intensities of few hundreds of Rayleigh. We can then calculate the $g$ factor following the same method as previously and derive a $g$ factor equal to $13.35 \mathrm{~s}^{-1}$. Therefore the upper limit for the column density of $\mathrm{Ca}$ atoms is equal to $1.4 \times 10^{9} \mathrm{Ca} \mathrm{cm}^{-2}$. Bida et al. (2000) measured the column density between $3.67 \times 10^{7} \mathrm{Ca}$ 


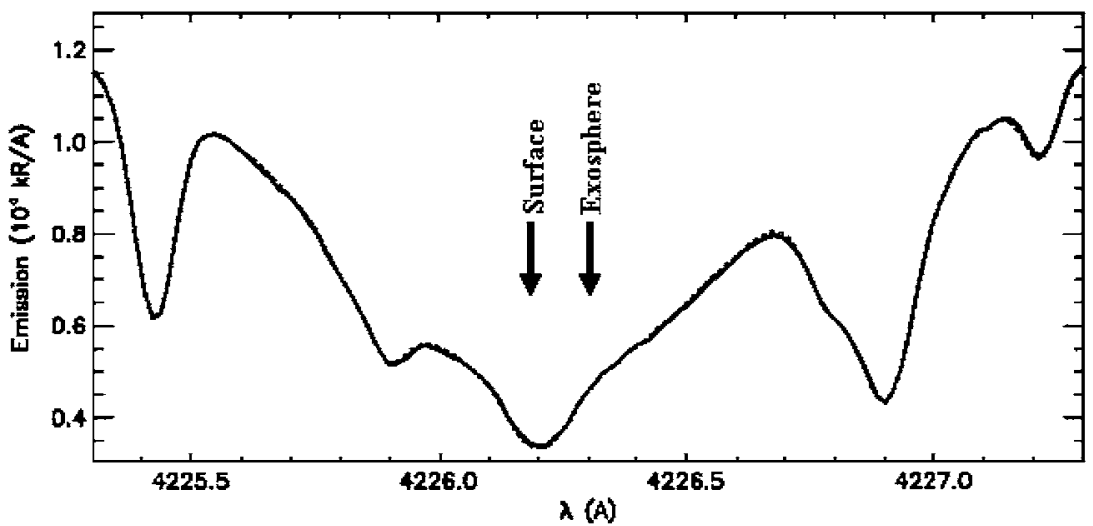

Figure 3 Spectrum measured by EMMI/NTT centered on the Ca $4227 \AA$ emission line. The positions of the exospheric line and of the solar absorption line reflected at Mercury's surface as expected from ephemeris are also indicated. A solar spectrum (BASS 2000, http://bass2000.obspm.fr) has been reduced in resolution, scaled to the measured spectra and plotted in dashed line.

Table 2

Abundances

\begin{tabular}{lcccc}
\hline \hline Species & Line $(\AA)$ & g-factor s $^{-1}$ & Intensity $(\mathrm{kR})$ & ${\text { Column Density }\left(\text { atoms cm }^{-2}\right)}^{\mathrm{A}}$ \\
$\mathrm{Al}$ & 3944.0060 & 0.786 & $<10.7^{\mathrm{a}}$ & $<14 \times 10^{9}$ \\
$\mathrm{Al}$ & 3961.5201 & 0.770 & $<6.0^{\mathrm{a}}$ & $<7.8 \times 10^{9}$ \\
$\mathrm{Na} \mathrm{D}_{2}$ & 5889.9510 & 23.13 & $1710 \pm 127^{\mathrm{a}}$ & $7.4 \pm 0.5 \times 10^{10}$ \\
$\mathrm{Na} \mathrm{D}_{1}$ & 5895.9242 & 13.74 & $1260 \pm 127^{\mathrm{a}}$ & $9.2 \pm 0.9 \times 10^{10}$ \\
$\mathrm{Ca}$ & 4226.728 & 13.35 & $<19^{\mathrm{a}}$ & $<1.4 \times 10^{9}$ \\
$\mathrm{Fe}$ & 3920.2578 & 0.170 & $<18^{\mathrm{b}}$ & $<1 \times 10^{11} \mathrm{Fe}$ \\
$\mathrm{Li}$ & 6707.761 & 56.38 & $<6^{\mathrm{b}}$ & $<4 \times 10^{7}$ \\
& 6707.912 & 84.64 & & \\
& 6708.072 & 28.27 & & $<5 \times 10^{10}$ \\
$\mathrm{Si}$ & 3906.629 & 0.31 & $<16^{\mathrm{b}}$ & \\
\hline
\end{tabular}

Notes.

${ }^{a}$ First night.

${ }^{\mathrm{b}}$ Average on both nights.

$\mathrm{cm}^{-2}$ and $1.8 \times 10^{8} \mathrm{Ca} \mathrm{cm}^{-2}$ (Bida et al. 2000) corresponding to an average zenith column density equal to $1.1 \times 10^{8} \mathrm{Ca} \mathrm{cm}^{-2}$, in good agreement with Killen et al. (2005).

In the same way, we can derive upper limits for the column density of $\mathrm{Fe}$ at $3920 \AA$, of $\mathrm{Li}$ at $6708 \AA$ and of $\mathrm{Si}$ at $3906 \AA$ during the first night. We found an upper limit for the emission brightness of these emission lines of 31, 8, and $27 \mathrm{kR}$ for $\mathrm{Fe}$ at $3920 \AA, \mathrm{Li}$ at $6708 \AA$, and Si at $3906 \AA$, respectively (and of $18 \mathrm{kR}, 6 \mathrm{kR}$, and $16 \mathrm{kR}$ when we average all spectra of both nights). These emission lines correspond then to upper limits of $2 \times 10^{11} \mathrm{Fe} \mathrm{cm}^{-2}, 5 \times 10^{7} \mathrm{Li} \mathrm{cm}^{-2}$ and $8 \times 10^{10} \mathrm{Si} \mathrm{cm}^{-2}$ tangent column densities in Mercury's exosphere during the first night of observation (to $1 \times 10^{11} \mathrm{Fe} \mathrm{cm}^{-2}, 4 \times 10^{7} \mathrm{Li} \mathrm{cm}^{-2}$, and $5 \times 10^{10}$ $\mathrm{Si} \mathrm{cm}^{-2}$ in an average during both nights).

\section{DISCUSSION AND CONCLUSION}

Our EMMI observations led to the marginal detection of $\mathrm{Al}$ exospheric lines both at $3944.0060 \AA$ and 3961.5201. This detection should be confirmed by further observations and can be used as an upper limit for this element in Mercury's exosphere.

If confirmed by further observations, using the lower bound of the column density for the aluminum measured by the EMMI and the upper limit on the Ca column density, we would therefore be left with a ratio between aluminum and calcium between 2 and 18 . Since both aluminum and calcium are refractory species, it is most probable that they are ejected from
Mercury's surface by energetic processes such as ion sputtering or meteoritic vaporization. Both these processes are efficient and roughly stoichiometric with respect to the relative abundance in Mercury's upper surface. Starting from a regolith composition close to that suggested by Morgan \& Killen (1997), that is with a regolith enriched in aluminum by less than $70 \%$ larger with respect to $\mathrm{Ca}$, Johnson \& Baragiola (1991) calculated a ratio of 4 between the $\mathrm{Al}$ and $\mathrm{Ca}$ exospheric abundances. This was derived using Hapke formulation of regolith sticking, ballistic lifetime, and ionization loss. In this model, the difference in mass is large enough to enrich the exosphere of the Moon in $\mathrm{Al}$ with respect to $\mathrm{Ca}$ which implies that a similar ratio would be found at the Mercury. This model also predicted an abundance in $\mathrm{Si}$ (which is also a refractory specie) six times larger than that of $\mathrm{Ca}$ which is therefore also in agreement with our upper limit for this specie, $5 \times 10^{10} \mathrm{Si} \mathrm{cm}^{-2}$. We also confirm the upper limits set by Sprague et al. (1996) for the Li abundance in Mercury's exosphere as being smaller than $4 \times 10^{7} \mathrm{Li} \mathrm{cm}^{-2}$ and provide the first upper limit for the exospheric iron as $10^{11} \mathrm{Fe} \mathrm{cm}^{-2}$ (see Table 2). The iron upper limit is interesting by the fact that Mercury surface is known to be depleted in ferrous iron (Vilas 1985). The content of $\mathrm{Fe}$ in the exosphere could possibly constrain, once the source processes are clearly understood, the regolith's composition. This could in turn permit to distinguish between differents scenario formations (Goettel et al. 1988). The Mercury Atmospheric and Surface Composition Spectrometer (MASCS) onboard the MESSENGER spacecraft, should be 
able to detect $\mathrm{Fe}$ exospheric line at $3719 \AA$, as well as many other species in the $115-600 \mathrm{~nm}$ spectral range $($ McClintock \& Lankton 2007).

However, this increase from surface to exosphere is not enough to reconcile our marginal measurements of $\mathrm{Al}$ with the typical column density for the $\mathrm{Ca}$ measured by Bida et al. (2000). Therefore, either the real abundance of Al in Mercury's exosphere is significantly below the values derived here, or the main sources of $\mathrm{Ca}$ and $\mathrm{Al}$ exospheric atoms are either not similar and in any case not stoichiometric with respect to the surface. In that later case, the value of the column density of $\mathrm{Al}$ in Mercury's exosphere derived from our marginal identification would suggest that either $\mathrm{Ca}$ or $\mathrm{Al}$ is not produced stoichiometrically from the surface into the exosphere or that the surface of Mercury should be significantly enriched in $\mathrm{Al}$ with respect to $\mathrm{Ca}$.

At the end, the presence of any atomic Al species in Mercury's exosphere should be associated with a population of $\mathrm{Al}^{+}$ions since the photo-ionization lifetime of an $\mathrm{Al}$ atom at Mercury distance to the Sun is typically of less than 5 minutes (Fulle et al. 2007). During its first flyby of Mercury, MESSENGER did not report the detection of such ionic species (Zurbuchen et al. 2008), even if their Figure 1 might suggest the existence of a population at the mass 27 .
We thank R.E Johnson and E. Lellouch for helpful comments on this paper during its preparation.

\section{REFERENCES}

Bida, T. A., Killen, R. M., \& Morgan, T. H. 2000, Nature, 404, 159 Broadfoot, A. L., Shemansky, D. E., \& Kumar, S. 1976, Geophys. Res. Lett., 3 , 577

Fulle, M., et al. 2007, ApJ, 661, L93

Goettel, K. A. 1988, in Mercury, ed. F. Vilas, C. Chapman, \& M. Matthews (Tucson, AZ: Univ. Arizona Press), 613

Johnson, R. E., \& Baragiola, R. 1991, Geophys. Res. Lett., 18, 2169

Killen, R. M., Bida, T. A., \& Morgan, T. H. 2005, Icarus, 173, 300

Kurucz, I. F., Brault, J., \& Testerman, L. 1984, National Solar Observatory Atlas No. 1 (Sunspot, NM: National Solar Observatory) NSO/Kitt Peak FTS data used here were produced by NSF/NOAO

McClintock, W. E., \& Lankton, M. R. 2007, Space Sci. Rev., 131, 481

Morgan, T. H., \& Killen, R. M. 1997, Planet. Space Sci., 45, 81

Morton, D. C. 2003, ApJS, 149, 205

Morton, D. C. 2004, ApJS, 151, 403

Potter, A. E., Killen, R. M., \& Morgan, T. H. 2007, Icarus, 186, 571

Potter, A. E., \& Morgan, T. H. 1985, Science, 229, 651

Potter, A. E., \& Morgan, T. H. 1986, Icarus, 67, 336

Sprague, A. L., Hunten, D. M., \& Grosse, F. A. 1996, Icarus, 123, 345

Vilas, F. 1985, Icarus, 64, 133

Zurbuchen, T. H., et al. 2008, Science, 321, 90 